\title{
Design of a new therapy to treat snake envenomation
}

This article was published in the following Dove Press journal:

Drug Design, Development and Therapy

18 June 2014

Number of times this article has been viewed

\section{Leyla Shahidi Bonjar}

Department of Pharmacology, College of Pharmacy, Kerman University of Medical Sciences, International Campus, Kerman, Iran
Correspondence: Leyla Shahidi Bonjar Department of Pharmacology, College of Pharmacy, Kerman University of Medical Sciences, International Campus, Haft Baagh Blvd,

Kerman, 76I, Iran

Tel +989133420125

Fax +98 34I 2120725

Email shahidileylaa@gmail.com
Abstract: The prospective removal of snake venoms from the blood of snake-bitten patients is discussed here. Opportune neutralization of killer antigens from the blood of poisoned victims is a vital treatment step. Delays may lead to death, or cripple the patient permanently. The present procedure describes the elimination of venom antigens of a wide range of snakes from the blood of such patients. Compared to conventional treatments, the treatment is administrable in the lack of proper antivenoms, expected to be more effective with less side effects, covers a vast range of snake venoms, minimizes contact of venoms with internal tissues and organs, is applicable in patients sensitive to serum injections, has a high chance of effectiveness because there is no need to identity the snake involved to administer its specific antibody, and is capable of universal application. The principal component to this approach is a "polyvalent venom antibody column" (PVAC), which selectively traps venom antigens from blood in an extracorporeal circuit while detoxified blood returns back to the patient's body. The PVAC is intended for removal of numerous snake venom antigens in a relatively simple procedure. Detoxification is performed under the supervision of trained personnel using simple blood-circulating machines in which blood circulates from patient to PVAC and back to the patient aseptically. The device acts as a biological filter that selectively immobilizes harmful venom antigens from poisoned blood. For effective neutralization, the PVAC provides a large contact surface area with blood. The PVAC's reactive sites would consist of carbon nanotubes, on which a vast spectra of venoms' antibodies are bonded to. In this extracorporeal detoxification process, nocent antigens conjugate with their antibodies and become immobilized, and are eliminated from the poisoned patient blood. Detoxification resuscitation is expected to take 2-3 hours, when the titers of venom antigens in the blood reach harmless levels, as confirmed by sampling of the blood and appropriate serological evaluations. If conventional antivenoms do not cover the entire spectrum of venom antigens in blood, rehabilitation would be a matter of a longer period; whilst the PVAC covers the widest range of antibodies to remove the broadest range of venom antigens, the rehabilitation period would be shorter since venom antigens have been removed from the body in a few hours duration. PVACs are to be biotechnologically engineered against a wide spectra of antigens present in the venoms of the dominant poisonous snakes for a defined geographical zone; ie, a country, part of a continent, or an entire continent. As a polyvalent column, the PVAC bears a sufficient amount of venom antibodies of all snakes that pose a threat in the region. PVAC treatment would have high applicability in cases where the patient is unconscious and/or the snake identity is not clear for administration of related antivenom medication. For opportune administration, research on the use of PVACs in emergency ambulances should receive special attention. Starting in situ detoxification, such ambulances would provide more efficient resuscitations to envenomed patients.

Keywords: venom, toxin, intoxication, detoxification, blood, polyvalent antibody 


\section{Introduction}

The venoms of most snakes are a mixture of toxins, enzymes, and smaller molecules. ${ }^{1-4}$ The makeup of venoms' components varies widely from species to species. This variability is the reason for the widely differing effects of snakebites. Venoms may cause the following reactions: neurotoxic (pre/ post/synaptic), cardiotoxic, myolytic, coagulant (anticoagulant), hemostatic (activating/inhibiting), hemorrhagic, and possibly directly nephrotoxic or hepatotoxic actions. ${ }^{5}$ From the standpoint of toxicology, snake venoms are divided into two broad classes: hemotoxic and neurotoxic. For instance Ophiophagous hannah (King Cobra) has predominantly neurotoxic venom while Crotalus adamanteus (Eastern diamondback rattlesnake) has predominantly hemotoxic venom. ${ }^{6}$ Hemotoxic venoms affect the patient's blood and organs, inducing a breakdown or inflammation in the body. Envenomation with hemotoxic bites is severely painful since breathing hurts and tissues start to die. In contrast, neurotoxic venoms impact the nervous system, causing everything from seizures to death. Neurotoxic envenomations are the most deadly snakebites. Even then there exists two different classes, no snake fits completely in one class, since many snakes bear both types in their venoms.

Because of the complex toxins and enzymes present in both venom categories, bites by venomous snakes can cause paralysis that may prevent breathing, internal bleeding disorders that can end up in fatal hemorrhaging, permanent kidney failure, and severe tissue damage that can cause severe disability that may result in limb amputation. Translocation of venom from the bitten site, via blood, to organs such as the liver, muscles, spleen, kidneys, lungs, heart, and brain produces toxicities that may lead to death. Urgent detoxification reduces side effects. The main treatment in conventional therapies includes intravenous injection of mono and/or polyvalent antivenom antibodies.

Many researchers have attempted in vitro detoxification of venoms such as: ionizing X-irradiation, ${ }^{7,8}$ gamma irradiation, ${ }^{9}$ ultraviolet light, ${ }^{10}$ controlled iodination, ${ }^{11,12}$ leaf extracts, ${ }^{13}$ and marine algae $;{ }^{14}$ however, none could be applied for detoxification in real situations in humans.

\section{World Health Organization (WHO) report on human casualties from snakebites and the global situation of snake antivenoms}

According to statistics released by $\mathrm{WHO},{ }^{15}$ about 5 million people are bitten each year by poisonous snakes which results in 2.5 million envenomations, at least 100,000 deaths, and 300,000 amputations and other permanent disabilities. According to the report countries face a critical global shortage of appropriately safe and effective snake antivenoms, mainly because of: poor regulations; marketing of inappropriate antivenoms; and loss of confidence in the available antivenoms by clinicians, public health officials, and patients. According to Carissa Etienne, WHO Assistant Director-General, ${ }^{15}$ proper antivenoms are not available to many countries. Many others implement antivenoms that may not react against their target snakes. Most of the victims are women, children and farmers from poor rural regions who do not have proper health services, and when bitten by snakes, they do not receive appropriate treatment.

\section{The proposal}

The proposed approach consists of the application of a "polyvalent venom antibody column" (PVAC) to neutralize a vast spectrum of snake venoms from the blood of victims bitten by poisonous snakes. The analogy is similarly applicable for other groups of venomous animals such as arthropods, reptiles, etc. The PVAC detoxifies circulating blood by using polyvalent antibodies that are effective against a wide range of venoms' antigens. In the course of resuscitation of envenomed patients, PVACs behave as the key unit in the relevant blood circulating machine. They perform "out of body detoxification" for agglutination of blood venoms, toxins, and harmful antigens to check them from returning back to the body.

In this method, the blood of a poisoned patient is processed during passage through the PVAC. The procedure set in the PVAC approach would be similar to the "nanogold detoxifying machine" designed and reported previously by myself ${ }^{16}$ or the "renal dialysis machine" used for patients with renal failure, ${ }^{17}$ but instead of a dialysis membrane, the machine would be equipped with a PVAC. PVACs would have large surface areas for direct contact with blood to immobilize venom antigens. Polyvalent venom antibodies (PVAs) would be conjugated on contact surfaces of the reaction platforms. PVAs should be specifically prepared against snake venom antigens of a designated geographical zone; ie, a country, part of a continent, or an entire continent. They should be prioritized for use in the zone they are designed for.

Passage of blood through the PVAC immobilizes venom antigens on stationary PVAs. Similarly to a renal dialysis machine, blood circulates from patient to PVAC 
and from there back to the patient in a closed circuit until the venom antigens are depleted enough from the blood. The venom antigen level in the blood returning to the body can be monitored by sampling and evaluation in appropriate serological tests. Use of a PVAC would improve the treatment outcome because of two criteria: 1) the PVAC diminishes venom antigens in the blood and minimizes their contact with internal vital organs, and 2) if a PVAC is used along with conventional antivenom therapeutics, since venom antigens deplete steadily, the efficacy of conventional therapies would be much enhanced. Such dual therapy will leave the patient with decreased chronic venom toxicity and minimal or no side effects. It would be a truly lifesaving device, especially in cases where the identity of the snake or arthropod involved is unclear and selection of the appropriate antivenom is a hard decision to make. A PVAC is single-use and would be appropriately disposed of after detoxification treatment. After sufficient testing in animal models, PVACs would gradually replace the conventional antivenom therapies in humans.

\section{PVAC approach}

Time is a key factor in lifesaving treatments for poisoned patients. When a victim gets access to proper medical assistance, he should first be treated according to conventional emergency protocols, in which administration of specific antivenoms has high priority. The PVAC would also be applied as combination therapy as soon as available. The PVAC could be present in equipped emergency ambulances to treat the patient in situ and on the way to the hospital. In emergency care units, the patient would also be treated for detoxification by PVAC. The PVAC should not interfere with any of the conventional treatments and resuscitations. The PVAC is supplemental to the conventional treatments. The period needed for PVAC treatment depends on the antigen titers in the blood before it returns back to the body of the patient; however, optimization studies in animals should set the procedure details. In cases where the patient is unconscious and the snake cannot be clearly identified for administration of proper antivenoms, PVAC treatments may have high applicability.

\section{PVAC structural detail and use}

As presented in Figure 1, a PVAC would be aseptically manufactured to have the following main features. The detoxification column has an inlet and an outlet for blood, a column cylinder which holds a stack of thousands of monovalent antibody plates (MAPs) around its center axis.

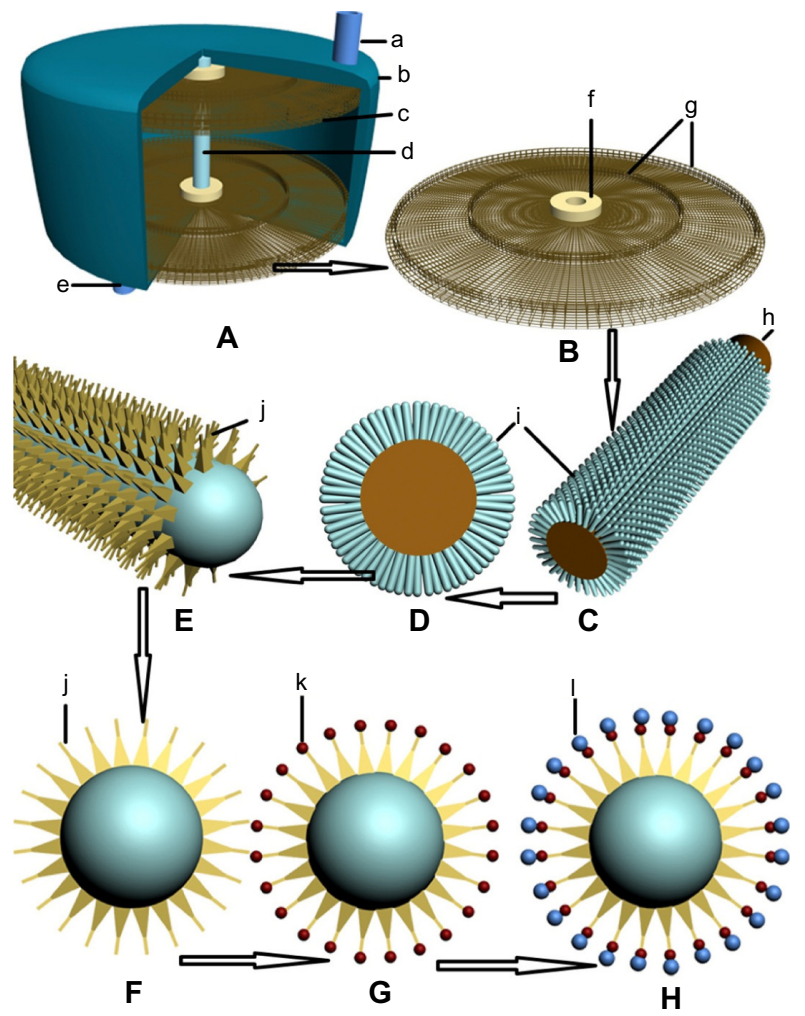

Figure I Schematic presentation of a polyvalent venom antibody column (PVAC) to trap and withdraw snake venom antigens from the blood of bitten patients in an extracorporeal circuit.

Notes: (A) Dissected PVAC, consisting of: (a) blood outlet, (b) column cylinder, (c) monovalent antibody plates (MAPs), (d) center axis which stacks MAPs in place, and (e) blood inlet. (B) A MAP manufactured from biocompatible organic polymers consisting of an extensive lattice, it bears ( $f$ ) a center spacer and $(g)$ lateral spacers to keep the plates apart. (C) The lattice frame is composed of subunits. (h) A lattice subunit. (D) Cross-section of a lattice subunit bearing millions of (i) carbon nanorods on its surface. (E) Surface topology of a carbon nanorods (antibody-antigen reaction platform). (F) Cross-section of a carbon nanorods harboring (j) antibody-conjugated moieties. (G) (k) Antibodies adhered to conjugated moieties. (H) (I) Venom antigens coagulated to their specific antibodies and immobilized from the circulating blood.

Each MAP consists of an extensive lattice of biocompatible organic polymers. MAPs harbor millions of carbon nanorods on their entire surfaces. Carbon nanorods, which act as "antibody-venom-antigen reaction platforms," are coated with monovalent antibodies via supportive intermediate moieties. The stacking of PVACs with MAPs takes place in a manufacturing company. The types and number of various MAPs to stack depends upon the spectrum of venom antigens of the regional snakes the PVAC is being assembled for. While the column is in action, each nocent antigen coagulates with its antibody on the appropriate MAP and would be immobilized thereafter.

Each MAP is engineered for a specific venom antigen that it is planned to immobilize. The number of MAPs per PVAC depends on the maximum titer of relevant antigens injected by each specific snake when it administers a bite to its human victim; however, information about venom antigen 
titers comes from venom toxicological studies. For an ideal performance, the number of specific MAPs per PVAC may be in the hundreds and the total number of different MAPs per PVAC may exceed several thousands. Assuming a $20 \mu \mathrm{m}$ thickness and $5 \mathrm{~cm}$ diameter for each MAP, and 20,000 MAPs per PVAC, the maximum height and volume of the column would be about $40 \mathrm{~cm}$ and $800 \mathrm{~cm}^{3}$, respectively. With such approximations, the column may have an internal volume to hold $900 \mathrm{~mL}$ of blood. The volume of the involved silicone connection tubes may not exceed $100 \mathrm{~mL}$. Thence, for an average-sized victim, the total volume of blood being processed at a given point of time would be about 1,000 mL. The flow rate may be $400-600 \mathrm{~mL} /$ minute, resulting in about 24-36 L of blood passing through the PVAC per hour. Considering a duration of 3 hours per treatment, the approximate volume of blood passing through the PVAC would be approximately 62-108 L. Under such circumstances, if the circulating machine and PVAC perform as needed, there exists a high chance of convenient extracorporeal detoxification of the bitten victims; however, the settings would be adjusted according to the appropriate investigations.

\section{Clinical significance}

Effective withdrawal of venom antigens from the blood of poisoned victims may be a lifesaving treatment. Any delay or failure in such a vital treatment may lead to death or cripple the patient for rest of his life. The main clinical significances of the PVAC that rationalize its applicability are as follows: 1) in the geographical zone that the PVAC is designed for, the PVAC is applicable without the need to determine the identity of the snake involved; 2) the PVAC is applicable whether conventional antivenoms are available or not; however, conventional therapies may be applied simultaneously while available; 3 ) the PVAC detoxifies the blood outside the body, hence it lowers venom contact with vital internal organs; 4) as a unique set, the PVAC is multifunctional, applicable against almost all poisonous snakes of a geographical zone that it has been designed for; 5) the PVAC can be used with conventional blood dialysis machines modified or designed for such application; 6) the PVAC reduces chronic venom toxicity; 7) the PVAC lowers the chance of many amputations and permanent disabilities resulting from delayed detoxification; 8) when optimized, the PVAC would be a ready-to-use device; 9) the PVAC has the potential to become an emergency appliance for emergency ambulances and emergency care units in hospitals; 10) since it is aseptically made for a single use, the PVAC reduces the chance of further infections; 11) if conventional antivenoms do not cover the entire spectrum of venom antigens in blood, rehabilitation would be a matter of a longer period; whilst the PVAC covers the widest range of antibodies to remove the broadest range of venom antigens, the rehabilitation period would be shorter since venom antigens would be removed from the body in a few hours duration; 12) the PVAC approach reduces immunoglobulin-triggering events (anaphylaxis), which in some patients are potentially life-threatening allergic reactions to antivenom injection. ${ }^{18,19}$

\section{Prospective difficulties, side effects, and remedies}

As proposed, PVAC treatment is performed in about a 3-hour session in which $60-80 \mathrm{~L}$ of the patient's blood circulates through the PVAC; however, similarly to hemodialysis, PVAC treatment may also be confronted with some problems and side effects. Side effects are generally proportionate to the volume and speed of blood removal. The probable difficulties (and remedies) may include: 1) Coagulopathy. Some snake venoms cause such a malady, which is uncontrolled internal or external bleeding. If left untreated, it may cause damage to internal organs and may be life-threatening. ${ }^{20}$ Reduced or absent clotting factors are administered along with PVAC therapy; eg, prothrombin complex concentrate, factor XIII, and fibrinogen. Based on the condition of the patient, the physician decides if this therapy should be given to treat bleeding that has already begun or to prevent bleeding from occurring. 2) Hypotension (the blood circulating machine can be programmed so that the right amount of blood is removed). 3) Muscle cramps (doctors are able to recommend some remedies). 4) Infection (proper care should be taken to perform the treatment aseptically to prevent infection). 5) Clotting (heparin is the most commonly used anticoagulant). 6) Itching, dry mouth, and anxiety (doctor's prescription would control them). However, the possible side effects of PVAC treatment would be less compared to hemodialysis since in PVAC treatment, no dialysis occurs and no electrolytes are exchanged, and treatment is initiated and managed by a specialized staff. 7) PVAC treatment may not be able to trap some neurotoxic venoms that are bound to neural synapses; however, that is a defectiveness of conventional antivenoms too. Such imperfection may contribute to ongoing symptoms and tissue damage. Repeating PVAC treatment plus infusion fluids may improve the situation. 8) Manufacture of high-quality venom-specific antibodies is a key feature in the development of PVAC treatment. It needs appropriate investments, collaboration of many fields of research, and development in several interdisciplinary sciences of medical engineering, 
nanotechnology, immunology, biochemistry, and emergency medicine to make the PVAC dream come true.

\section{General considerations in selection of venom sources for production of polyvalent antivenoms of PVACs}

Since PVACs are to be equipped with most of the antivenoms of the geographical zone that they are designed for, the careful choice of venom sources is an important issue. The antigenic makeup of venoms has the highest impact in antivenom production and efficacy. To ensure the representation of all venom antigens required for the antivenoms in the PVACs, all known factors affecting variability of venoms should be taken into consideration when selecting snake sources for antivenom production. Factors affecting venom variability arise at several levels: interfamily, intergenus, interspecies, intersubspecies and intraspecies, geographical variation, seasonal variation, diet, habitat, wild or captive specimens, age-dependent change, and sexual dimorphism. ${ }^{5}$ In other words, to ensure representation of most venom antigens required in the polyvalent antivenoms of PVACs, production of antivenoms must utilize an understanding of such variability when selecting sources for venoms.

\section{Further prospective}

PVAC treatment combines nanotechnology and medicine, and could be used for applications other than the treatment of venomous bites. In this regard, research with a similar approach should arise for the removal and withdrawal of systemic toxins, antigens, and microorganisms from infected patients' blood through the extracorporeal immobilization of a wide range of systemic maladies. PVACs would have stationary antibodies for removal of the target antigens. It is clear that removal of harmful antigens would lead to improvement of patients' health; thereafter, the general performance of the patients would improve and conventional therapies would work more efficiently. Examples of such rehabilitations are as follow: 1) bacterial sepsis not responsive to conventional antibiotic therapies, eg, coagulase-negative Staphylococcus strain and Serratia liquefaciens, which may lead to death in less than 25 hours; ${ }^{21}$ 2) viral blood infections, eg, viral hepatitis $\mathrm{B}$ and $\mathrm{C}$ and human immunodeficiency virus; 3) botulism; 4) tetanus antigens and related toxins; 5) blood fungal infections, eg, septicemia with Candida albicans; 6) biological wars in which sepsis and microbial blood invasions occur; 7) in cases of Alzheimer's and Parkinson's diseases, if their causative or specific antigens are determined, regular or occasional removal of such antigens from the blood and/or cerebrospinal fluid may improve the patient's health and slow down the disease progression; and 8) to enhance blood transfusion safety regarding undesirable residues of some antigens, such columns may be used as prophylactic measures prior to transfusions of blood derivatives. Each of the proposed approaches should be evaluated for their applicability; however, optimization studies in animals should always pave the way for future human applications.

Conclusively, it should be specified that antibody columns are not to replace the conventional treatments; however, their selective removal of the target antigens from blood would make conventional therapies more effective. I hope that expanded application of the PVAC approach will reduce human casualties.

Prior to PVAC implementation in humans, it should be evaluated in animals; however, future investigations would determine its applicability in each of the abovementioned areas. Animal experiments should mimic human cases as closely as possible including time of access to treatment. Five treatments should be included in studies including: 1) healthy untreated, 2) envenomed, treated with conventional antivenoms, 3) envenomed treated with PVAC alone, 4) envenomed, treated with combination therapy (conventional plus PVAC), and 5) envenomed, receiving neither of the therapies. Treated animals should receive meticulous assessment throughout their lifetime to reveal probable side effects of PVAC treatment on vital internal organs such as the liver, muscles, spleen, kidneys, lungs, heart, and brain. Special attention should be placed on investigating hematological toxicity (blood), hepatotoxicity (liver), and nephrotoxicity (kidney) responses. The effectiveness of PVAC treatment in animal investigations would then allow for the use of PVACs on humans. When proved efficient either as stand alone or in combination therapy, then PVAC treatment could be used as a procedure to assist many envenomed patients. It should be emphasized that since PVACs encompass the widest spectrum of snakes of wide geographical regions, it may be the main choice in developing countries and rural zones where highquality venom-specific antibodies are lacking.

\section{Conclusion}

It is anticipated that PVACs will bear efficacious treatment of bite victims and reduce toxicity and side effects of snake envenomation. The PVAC approach, in combination with conventional therapies, would improve the outcome of detoxification and the well-being of treated patients. Importantly, this combination therapy would help to protect the patient's kidneys and internal organs more effectively. 
The application of PVAC treatment, if not feasible in all suggested areas, holds great promise to effectively improve the outcome of patients whose health is threatened by the invasion of deleterious antigens in their blood. PVAC treatment in combination therapy may shorten recovery periods since venom antigens are trapped and neutralized simultaneously in both intracorporeal and extracorporeal routes. In other words, in conventional therapies, the antibody-antigen complex remains internal, which takes a longer period to disintegrate, while in PVAC therapy such a period is omitted. Having such peculiarities, PVACs may create new approaches to the management of blood infections and treatment protocols in some diseases. Because of its performance, the PVAC may justify its presence as a necessary device in emergency and intensive care units in hospitals. The PVAC is anticipated to reduce the time, effort, and expense in blood detoxification therapies. I believe controlled application of the PVAC prevents many human casualties which conventional methods alone may not.

I believe that like other new medical devices, the PVAC therapeutic methodology would improve through interdisciplinary cooperation of scientists. PVAC achievements will set a smile on the faces of remediated patients, and the physicians and personnel who strive to bring health back to their patients.

\section{Acknowledgments}

I would like to dedicate this paper to those patients who would be resuscitated with the PVAC and get back to their families and living normal healthy lives; and also I dedicate it to the physicians and scientists who attempt to bring this desire to reality.

\section{Disclosure}

The author reports no conflicts of interest in this work.

\section{References}

1. Koh DC, Armugam A, Jeyaseelan K. Snake venom components and their applications in biomedicine. Cell Mol Life Sci. 2006;63(24): 3030-3041.

2. Baride RM, Jain SD, Gaitonde BB. Biochemical studies on the toxoids of venoms of poisonous Indian snakes. Indian J Med Res. 1980;72: 571-576.

3. Tu AT. Overview of Snake Venom Chemistry. Adv Exp Med Biol. 1996;391:37-62.
4. Hayes WK. Research on Biological Roles and Variation of Snake Venoms [webpage on the Internet]. Loma Linda: Loma Linda University; 2014. Available from: http://www.llu.edu/public-health/ ebs/hayes/research-c-venom.page. Accessed March 14, 2014.

5. Chippaux JP, Williams V, White J. Snake venom variability: methods of study, results and interpretation. Toxicon. 1991;29(11):1279-1303.

6. Venom. Venom types [webpage on the Internet]. Reptilis.net; 2012. Available from: http://reptilis.net/serpentes/venom.html. Accessed March 14, 2014.

7. Rogero JR, Nascimento N. Detoxification of snake venom using ionizing radiation. J Venom Anim Toxins. 1995;1(1):7-10.

8. Flowers HH. Effects of X-irradiation on the antigenic character of Agkistrodon piscivorus (cottonmouth moccasin) venom. Toxicon. 1966;3(4):301-304.

9. Flowers HH. Effects of X-irradiation on the antigenic character of Agkistrodon piscivorus (cottonmouth moccasin) venom. Toxicon. 1966;3:301-304.

10. Tejasen P, Ottolenghi A. The effect of ultra-violet light on the toxicity and the enzymatic and antigenic activities of snake venom. Patol Pol. 1970;21(3):225-233.

11. Daniel JP, Heneine LG, Tavares CA, Nascimento MC, Heneine IF. Generation of protective immune sera by Crotalus durissus terrificus venom detoxified by controlled iodination. Braz J Med Biol Res. 1987;20(6):713-720.

12. Rogero JR, Nascimento N. Detoxification of snake venom using ionizing radiation. J Venom Anim Toxins. 1995;1(1).

13. Abubakar MS, Sule MI, Pateh UU, Abdurahman EM, Haruna AK, Jahun BM. In vitro snake venom detoxifying action of the leaf extract of Guiera senegalensis. J Ethnopharmacol. 2000;69(3):253-257.

14. Vasanthi HR, Jaswanth A, Krishnaraj V, Rajamanickam GV, Saraswathy A. In vitro snake venom detoxifying action of some marine algae of Gulf of Mannar, south-east coast of India. Phytother Res. 2003;17(10):1217-1219.

15. WHO highlights critical need for life-saving antivenoms [webpage on the Internet]. Geneva: World Health Organization; 2014. Available from: http://www.who.int/mediacentre/news/notes/2010/ antivenoms_20100504/en/. Accessed March 14, 2014.

16. Shahidi Bonjar L. "Nanogold detoxifying machine" to remove idle nanogold particles from blood stream of cancer patients treated with antibody-nanogold therapeutics. Med Hypotheses. 2013;80(5): 601-605.

17. Joe S. How Does a Dialysis Machine Work? [webpage on the Internet]. Denver: DaVita HealthCare Partners Inc.; 2014. Available from: http://www.davita.com/treatment-options/hemodialysis/ in-center-hemodialysis/how-does-a-dialysis-machine-work?/t/5596. Accessed March 14, 2014.

18. Brown AF. Anaphylactic shock: mechanisms and treatment. $J$ Accid Emerg Med. 1995;12(2):89-100.

19. Bjornsson HM, Graffeo CS. Improving Diagnostic Accuracy of Anaphylaxis in the Acute Care Setting. West J Emerg Med. 2010;11(5): 456-461.

20. Rojnuckarin P. Snakebite-induced coagulopathy and bleeding disorders. In: Kini RM, Clemetson KJ, Markland FS, McLane MA, Morita T, editors. Toxins and Hemostasis: From Bench to Bedside. Berlin: Springer; 2010:699-710.

21. Brecher ME, Hay SN. Bacterial contamination of blood components. Clin Microbiol Rev. 2005;18(1):195-204. 


\section{Publish your work in this journal}

Drug Design, Development and Therapy is an international, peerreviewed open-access journal that spans the spectrum of drug design and development through to clinical applications. Clinical outcomes, patient safety, and programs for the development and effective, safe, and sustained use of medicines are a feature of the journal, which has also been accepted for indexing on PubMed Central. The manuscript management system is completely online and includes a very quick and fair peer-review system, which is all easy to use. Visit http://www.dovepress.com/testimonials.php to read real quotes from published authors.

Submit your manuscript here: http://www.dovepress.com/drug-design-development-and-therapy-journal 